US Army Corps

of Engineers ${ }_{\circledast}$

Engineer Research and

Development Center

Engineered Resilient Systems

\title{
TradeAnalysis Visualization Comparisons
}

Kenneth D. Niles, Joseph E. Jabour, Joshua Q. Church,

August 2019 and Timothy W. Garton

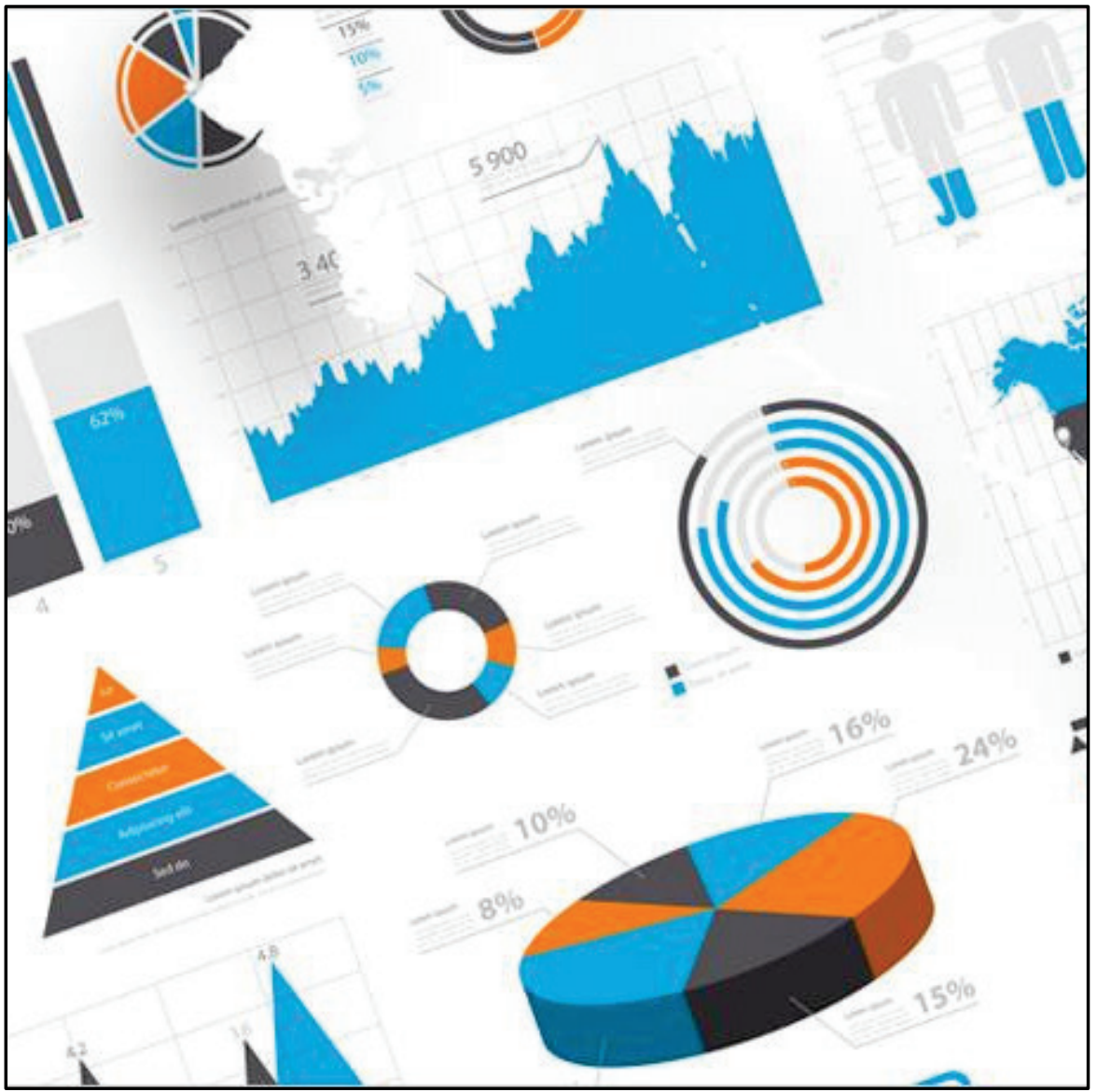


The U.S. Army Engineer Research and Development Center (ERDC) solves the nation's toughest engineering and environmental challenges. ERDC develops innovative solutions in civil and military engineering, geospatial sciences, water resources, and environmental sciences for the Army, the Department of Defense, civilian agencies, and our nation's public good. Find out more at www.erdc.usace.army.mil.

To search for other technical reports published by ERDC, visit the ERDC online library at http://acwc.sdp.sirsi.net/client/default. 


\section{TradeAnalysis Visualization Comparisons}

Kenneth D. Niles, Joseph E. Jabour, Joshua Q. Church, and Timothy W. Garton

U.S. Army Engineer Research and Development Center (ERDC) Information Technology Laboratory (ITL) 3909 Halls Ferry Road

Vicksburg, MS 39180-6199

Final Report

Approved for public release; distribution unlimited.

Prepared for Headquarters, U.S. Army Corps of Engineers

Washington, DC 20314-1000

Under Engineered Resilient Systems Program, Data Analytics Work Package, Collaborative Tradespace Analytics Work Unit 92L5D8 


\section{Abstract}

The objective of this report is to identify available tools that satisfy the customer's needs instead of recreating a tool that already exists. With the vast amount of data analysis and visualization tools on the market, it can be difficult to choose the correct tool for any given purpose. It should be noted that the goal of this report is not to determine the best data analysis and visualization tool, but rather to inform readers about specific factors each tool offers. The TradeAnalysis team (in support of the Engineered Resilient Systems Decision Support Tradespace Analytics team) achieved this goal by first creating a list of factors to objectively compare each tool. Once the factors were determined, each team member was tasked to find viable tools on the market capable of generalizing solutions to customer's requirements. Once the research was completed, the team gathered the data and compiled this report to compare the advantages and disadvantages of the tools. 


\section{Contents}

Acrongms and Abbreviations

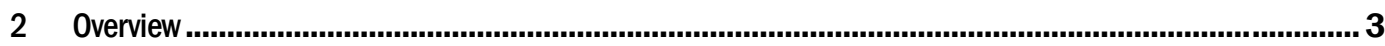

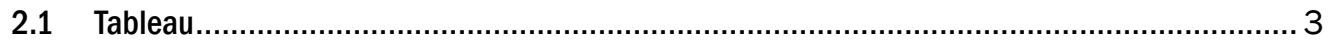

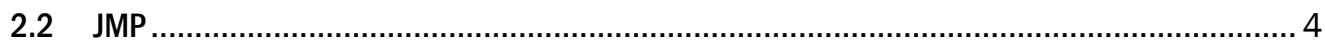

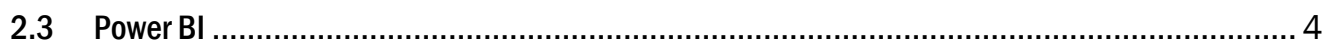

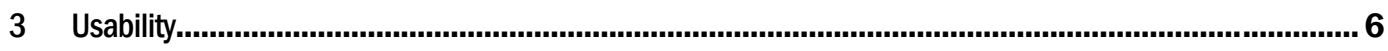

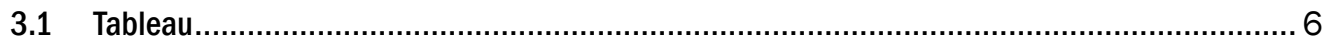

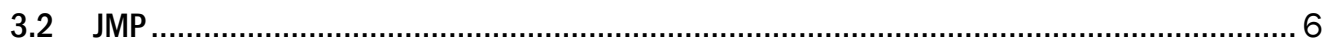

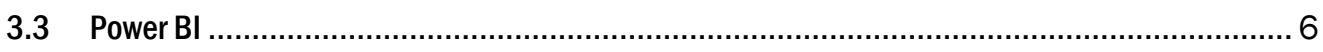

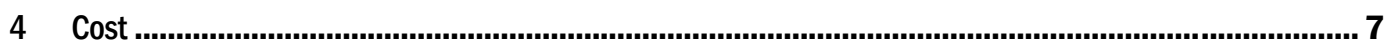

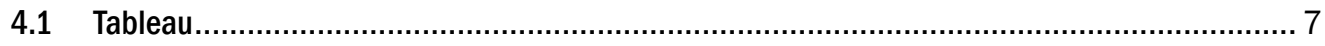

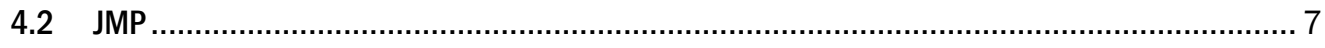

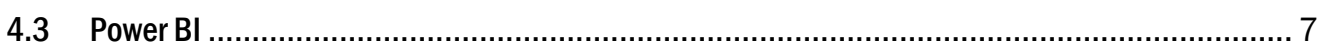

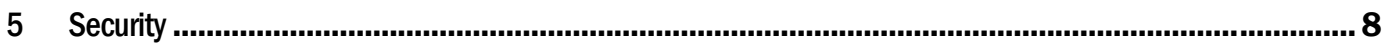

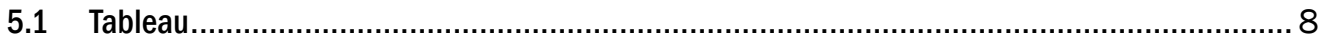

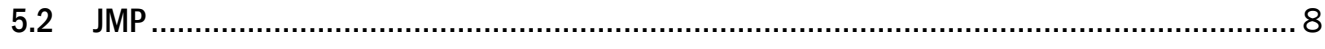

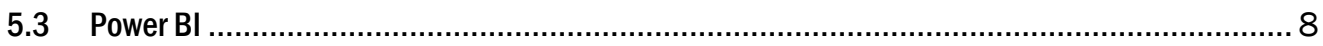

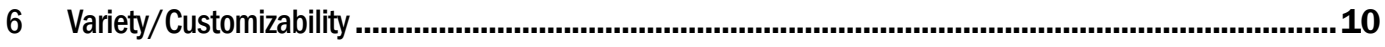

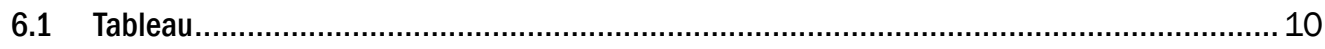

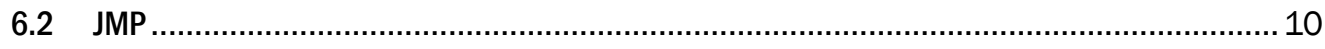

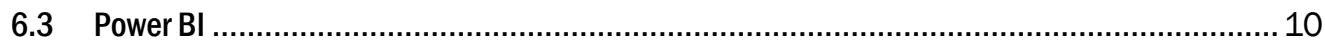

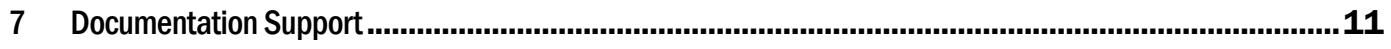

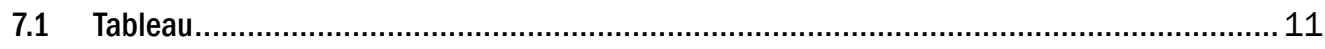

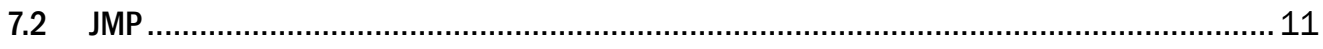

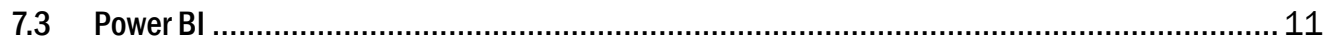

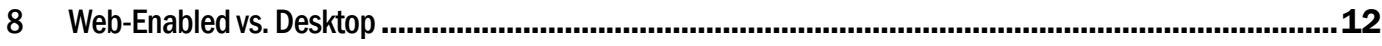

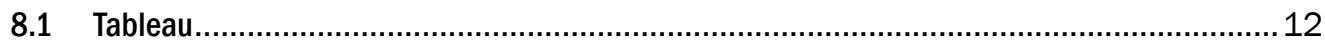

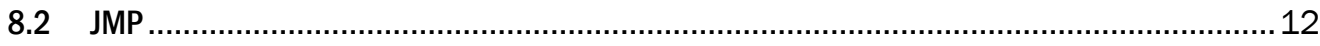




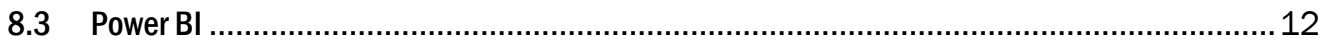

9 Developer Support.........................................................................................................................13

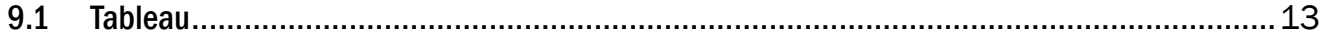

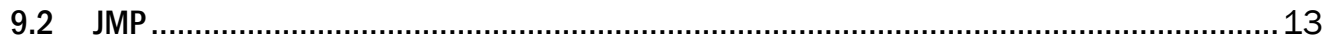

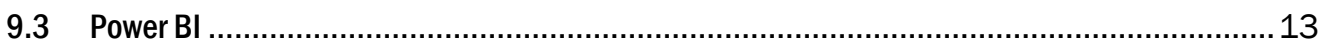

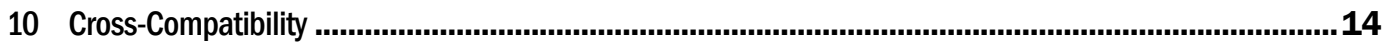

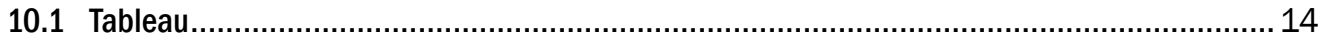

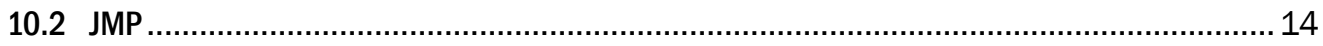

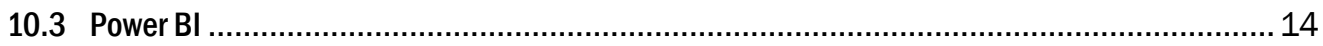

11 Efficiency with Large Data ...................................................................................................15

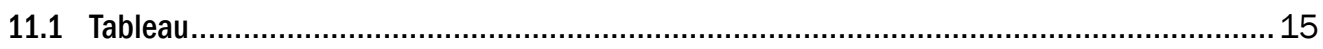

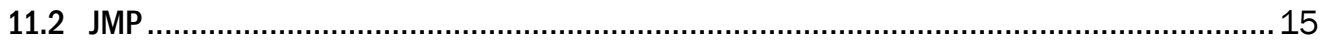

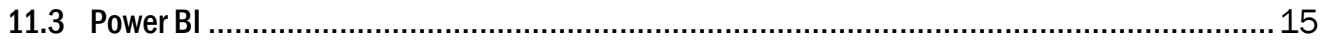

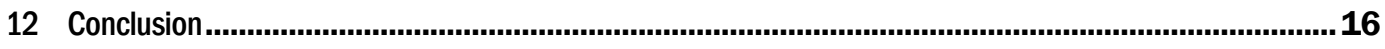

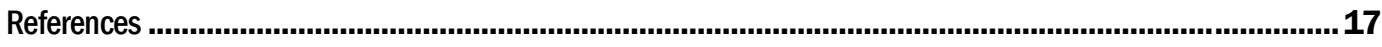

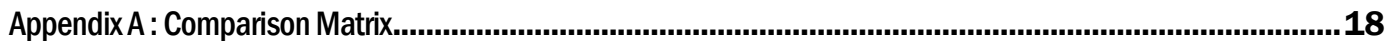

Report Documentation Page 


\section{Preface}

This report is a deliverable product under the Engineered Resilient Systems (ERS) Program, Data Analytics Work Package, Collaborative Tradespace Analytics Work Unit 92L5D8. Dr. Owen J. Eslinger was the Program Manager, and Dr. Robert M. Wallace was the lead Technical Director of the ERS program.

The work was performed by the Scientific Software Branch (SSB) and Computational Analysis Branch (CAB) of the Computational Science and Engineering Division (CSED), Engineer Research and Development Center (ERDC), Information Technology Laboratory (ITL), Vicksburg, MS.

At the time of publication, Mr. Timothy Dunaway was Chief, SSB and Dr. Jeffrey L. Hensley was Chief, CAB. Dr. Jerrell R. Ballard was Chief, CSED. The Deputy Director of ITL was Ms. Patti S. Duett and the Director was Dr. David A. Horner.

COL Ivan P. Beckman was Commander of ERDC, and Dr. David W. Pittman was the Director. 


\section{Acronyms and Abbreviations}

\begin{tabular}{|c|c|}
\hline Term & Meaning \\
\hline $2 \mathrm{D}$ & Two-Dimensional \\
\hline 3D & Three-Dimensional \\
\hline AAD & Azure Active Directory \\
\hline $\mathrm{BI}$ & Business Intelligence \\
\hline $\mathrm{CAB}$ & Computational Analysis Branch \\
\hline $\mathrm{CPU}$ & Central Processing Unit \\
\hline CSED & Computational Science and Engineering Division \\
\hline CSV & Comma Separated Values \\
\hline ERDC & Engineer Research and Development Center \\
\hline ERS & Engineered Resilient Systems \\
\hline GB & Gigabytes \\
\hline $\mathrm{GHz}$ & Gigahertz \\
\hline HTML & Hypertext Markup Language \\
\hline ISAE & International Standards for Assurance Engagements \\
\hline ITL & Information Technology Laboratory \\
\hline JSL & JMP Scripting Language \\
\hline MATLAB & Matrix Laboratory \\
\hline OS & Operating System \\
\hline RAM & Random Access Memory \\
\hline SAML & Security Assertion Markup Language \\
\hline SAS & Statistical Analysis Software \\
\hline SOC & Service Organizational Control \\
\hline sox & $\begin{array}{l}\text { Sarbanes-Oxley Compliance, in reference to the } \\
\text { Sarbanes-Oxley Act of } 2002\end{array}$ \\
\hline SSB & Scientific Software Branch \\
\hline SSL & Secure Sockets Layer \\
\hline SQL & Structured Query Language \\
\hline TLS & Transport Layer Security \\
\hline WFE & Web Front End \\
\hline
\end{tabular}




\section{Introduction}

\subsection{Background}

This report is a comparison of available custom and off the shelf tools that provide rapid visualization development for tradespace analytics and decision support. Available tools have been defined, and the capabilities of each tool compared and contrasted to assist in future software developmental decisions.

\subsection{Objectives}

The objective of this report is to address customer's data analysis and visualization requirements. The first step was to determine which features would satisfy the needs of the customers. Each member of the TradeAnalysis team (in support of the Engineered Resilient Systems Decision Support Tradespace Analytics team) constructed a list of features to be considered. Once the team members gathered their data, the following list was compiled: usability, cost, security, variety and customizability, documentation support, web-enabled vs. desktop, developer support, cross-compatibility, and efficiency with large data.

\subsection{Approach}

Once the list of essential features was established, each team member was tasked to find viable tools on the market capable of generalizing solutions to customer's requirements. The team discussed their findings and narrowed the list to the following three industry-standard tools: Tableau, Statistical Analysis Software (SAS) JMP, and Microsoft Power Business Intelligence (BI).

These three tools were then distributed amongst the team and each member was tasked to research the tool assigned to them. After this stage was complete, the team members created a report analyzing how each factor applied to their assigned tool. The reports were merged together to create both a comparison matrix, and a summary of each factor included in the matrix. 


\subsection{Scope}

The scope of this report will focus directly on available custom and off the shelf tools available to provide rapid visualization support for tradespace analytics and decision support. 


\section{Overview}

This section covers a high-level overview of the various aspects for each of the tools researched for this effort. Appendix A contains a comparison matrix of the information.

\subsection{Tableau}

Tableau is one of the industry-leading data visualization tools, blending both data science and BI into one software suite. Tableau allows the user to perform real-time data analysis and filter down the data to a more human-readable context (Jain 2017). The following three versions of Tableau are available: Viewer, Explorer, and Creator. Each version of Tableau includes Tableau Server, which is an on-premises, or public cloud server used for company-wide projects.

Tableau Viewer is designed for the user who requires read-only permissions to view dashboards created within Tableau. The user is given the ability to interact with the visualizations and dashboards, download visualizations as images, and add custom comments to the dashboard or visualizations (Tableau Software n.d.). The user with viewer privileges cannot create or modify any existing dashboards, but can interact with the created visualizations (Tableau Software n.d.).

Tableau Explorer is designed for the user who requires the abilities of the Viewer features with additional functionality to create and edit dashboards. Along with the features outlined in the Viewer license, the user can also download the full data, create and share custom views, create and publish new workbooks with existing data sources, and manage users and permissions (Tableau Software n.d.).

Tableau Creator is designed for the user who requires the features of both the Viewer and Explorer with additional governance, author, and preparation features. With the Creator license, the user has the ability to create, edit, and modify data flows, export data in various formats, and govern server administration (Tableau Software n.d.). As an administrator in Tableau, the user can monitor and scale hardware and track and manage content, users, licenses. 


\subsection{JMP}

JMP is a high-end data analysis and visualization tool. It is designed to give the user the ability to explore and view data with robust customizability. JMP aids the user by offering a wide variety of visualizations to graphically represent statistical data.

JMP offers multiple versions to fit the user's specific needs: JMP, JMP Pro, JMP Clinical, JMP Genomics, and JMP Graph Builder for iPad. Due to the requirements of this report, the focus is on JMP and JMP Pro.

The standard JMP package supports many capabilities, such as data cleaning, custom scripting, and data analysis. The data cleaner takes care of most entry errors, missing values, and other inconsistencies. JMP Scripting Language (JSL) allows the user to extend the functionality of the features within the software. The data analysis feature allows the user to filter down and find additional insight with the use of regression, distribution fitting, and other analysis techniques (JMP Statistical Discovery n.d.).

JMP Pro includes all the features found in JMP. In addition, JMP Pro also offers advanced features, such as predictive modeling with cross validation and modern statistical modeling. JMP Pro employs additional techniques to perform modern statistical modeling on a variety of data (JMP Predictive Analytics Software n.d.).

\subsection{Power BI}

Power BI is Microsoft's solution to business intelligence software. Based on Microsoft Excel add-ins, Power BI was created with the intention of being an easy way for companies to view their data and optimize their business practices.

Power BI comes in the following three versions: Desktop, Pro, and Service. There is a Premium purchase option available; however, this option is simply a bulk purchase of Pro licenses with a copy of Power BI Report Server (Microsoft Pricing for Power BI n.d.).

Power BI Desktop is the standard version of Power BI. It comes with nearly every feature offered by the Power BI service, including all available graphs, charts, and data manipulation tools. 
Power BI Pro includes all features available in Power BI Desktop, with the additional ability to share presentations with users on the Power BI online service or using the Power BI Report server (Microsoft Bring your Data to Life n.d.).

Power BI Service is an online-only module that allows the user to upload a dataset and manipulate it using all existing visualizations featured in the Power BI desktop application variants. Power BI Service is also the means by which Power BI users can share their presentations. The drawback of Power BI Service is its lack of direct data manipulation. While the user can create visualizations using the uploaded data, the raw data itself cannot be edited from within Power BI Service, as it can with its desktop application counterpart. 


\section{Usability}

This section covers the level of difficulty each tool presents from the viewpoint of a new user with limited to no experience.

\subsection{Tableau}

The Tableau interface is intuitive and user-friendly. It offers many features that allow the user to quickly upload data and highlight relationships. The user can easily apply basic visualizations to the uploaded data. In addition, the user can create advanced visualizations with the help of the expansive documentation offered by Tableau.

\subsection{JMP}

The JMP interface displays much of its functionality on the screen at the same time. Although these elements are easily accessible, it can overwhelm a new user with too many options upfront. This software seems to be tailored towards experienced data analytic users. The style of the interface looks archaic as opposed to its newer competitors, but the consistent look allows previous users to quickly adapt to the newer versions of the software.

\subsection{Power BI}

The Power BI interface offers a more modern approach to business intelligence data analysis tools. The intuitive drag-and-drop functionality allows the user to apply various visualization options with the uploaded dataset, allowing for custom-built and shareable data visualization dashboards. The user can quickly connect different datasets via Microsoft's Office suite or various Structured Query Language (SQL) databases. 


\section{Cost}

This section covers the pricing for each of the researched tools.

\subsection{Tableau}

Tableau is comprised of the following three versions: Viewer, Explorer, and Creator. A Viewer license has an upfront cost of $\$ 144.00$ USD/user/year. An Explorer license is $\$ 420.00$ USD/user/year. A Creator license is $\$ 840.00$ USD/user/year (Tableau Software Pricing n.d.).

\subsection{JMP}

JMP is comprised of many versions, however, this report will cover the following versions: JMP and JMP Pro. The basic JMP package has an upfront cost of \$1,785.00 USD/unit/year (JMP Multichannel marketing n.d.). The JMP Pro package has an upfront cost of $\$ 14,900.00$ USD/unit/year (JMP Pro Multichannel marketing n.d.).

\subsection{Power BI}

Power BI is comprised of the following three versions: Desktop, Pro, and Premium. Power BI Desktop is a free-to-use program (Power BI Premium calculator n.d.). Power BI Pro has an upfront cost of $\$ 119.88$ USD/user/year (Power BI Premium calculator n.d.). Power BI Premium contains the features of the Pro version and includes Power BI Report server. The upfront cost is $\$ 119.88 \mathrm{USD} / \mathrm{user} /$ year with an additional cost of $\$ 5,9940.00 /$ node/year (Microsoft Pricing for Power BI n.d.). The number of nodes included in the purchase is determined by the number of users specified in the purchase. 


\section{Security}

This section covers the security features for each of the researched tools.

\subsection{Tableau}

Tableau focuses on the following four security components: authentication, authorization, data security, and network security.

Tableau supports a variety of authentication methods including Active Directory, Kerberos, OpenId Connect, Security Assertion Markup Language (SAML), trusted tickets, certificates, and built-in local authentication. Tableau servers offer administrative privileges and rolebased access control, which allows certain users to view or edit specific projects. This feature enables users to collaborate on various projects (Tableau Software Platform Security n.d.).

Tableau offers a flexible security model for database security, which can blend the user's existing security practices with those of Tableau. Tableau is able to meet the data security standards of the Sarbanes-Oxley Compliance (SOX), Service Organizational Control (SOC), and International Standards for Assurance Engagements (ISAE) (Tableau Software Platform Security n.d.).

The final component in Tableau security is network security. To protect against unwanted networks, Tableau encrypts all transmissions using Secure Sockets Layer (SSL) and Transport Layer Security (TLS) (Tableau Software Platform Security n.d.).

\subsection{JMP}

JMP does not have any supporting documentation about security. Since JMP is a desktop-based tool, the security depends on the environment it is hosted on.

\subsection{Power BI}

Microsoft Azure is the foundation for Power BI's security platform. Azure is Microsoft's leading cloud computing service. Power BI deploys with two clusters, Web Front End (WFE), and Back-End. WFE facilitates the process of establishing the initial connection and authentication between 
the user and the service. The Back-End cluster facilitates the remaining connections and interactions (Iseminger et al. 2018).

Within the WFE cluster, Power BI employs different methods to properly authenticate the user and deliver content securely. Among these is the Azure Traffic Manager, which Power BI uses to direct user traffic to the correct datacenter. This allows for secure download of content files requested by the user. The distribution is handled by the Azure Content Delivery Network, which can efficiently handle the content based on geographical location. For user identity management and storage, Power BI leverages Azure Active Directory (AAD) (Iseminger et al. 2018).

The Back-End cluster handles the primary features of Power BI, such as the visualizations, data storage, etc. The Gateway Role handles all connections with the Back End, and links all the authentication, authorization, and protection roles for the primary Power BI services. Azure BLOB is used to manage data storage, and Azure SQL Database is used to store metadata (Iseminger et al. 2018). 


\section{Variety/Customizability}

This section covers the visualization variety and customizability offered by each of the researched tools.

\subsection{Tableau}

Tableau offers a wide variety of two-dimensional (2D) visualizations, ranging from scatter plots to box-whisker plots. Tableau allows the user to create custom R and Python scripts for graph customization. Currently, Tableau does not support three-dimensional (3D) visualizations and there is no supporting evidence for $3 \mathrm{D}$ visualizations in the future.

\subsection{JMP}

JMP offers a mixture of both 2D and $3 \mathrm{D}$ visualizations, ranging from scatter plots to contour profilers. JMP is compatible with other analysis tools, such as Matrix Laboratory (MATLAB) and specialized R libraries. JMP also allows the user to develop custom scripts using the JMP Scripting Language (JSL).

\subsection{Power BI}

Power BI offers a mixture of both 2D and 3D visualizations, including bar graphs, line charts, pie charts, and maps to view the geographical location affected by the underlying data. Power BI incorporates the option to download official and community-made visualizations from the Microsoft marketplace. The user can also create their own visualizations using the Power BI Custom Visual tool. In addition, Power BI includes the Power Query, this allows the user to perform data manipulation within the software without the assistance of an outside tool. 


\section{Documentation Support}

This section covers the availability of online documentation for each of the researched tools.

\subsection{Tableau}

Tableau is well documented and easily digestible. The documentation is officially supported and backed by a rich user community that supplies hundreds of online resources. The documentation ranges from basic setup assistance to designing advanced data analysis and visualization dashboards. In support of the documentation, Tableau also offers inperson and online classes to help provide an interactive learning experience.

\subsection{JMP}

JMP offers extensive, highly-detailed documentation for all the features included in the software, however, finding a specific use-case within the documentation is difficult. JMP also has a dependable community that provides many additional online resources.

\subsection{Power BI}

Power BI has extensive documentation on the Microsoft website. The documentation includes detailed tutorials on various aspects of Power BI, such as installation, setup, and features within the application. In addition to official documentation, there are community forums dedicated to Power BI that allows users to assist one another and answer potential questions not covered in the official documentation. 


\section{Web-Enabled vs. Desktop}

This section covers the online and desktop variations of each of the researched tools.

\subsection{Tableau}

Tableau is available as a desktop, online, and mobile application. The core features of Tableau exist in the desktop application, but this edition leverages the resources of the device it is installed on. The online application prevents the user from having to configure servers, manage software upgrades, or scale hardware capacity. With their analysis platform hosted in the cloud, the user can quickly share interactive visualizations and accurate data with coworkers and customers from a browser or on the go with Tableau's mobile application.

\subsection{JMP}

JMP is only supported as a desktop application, but it allows the user to export projects to Hypertext Markup Language (HTML) files to view the project in a web browser with the same interactive capabilities.

\subsection{Power BI}

The desktop edition is composed of the following two versions: Free (simply referred to as Desktop), and Pro. Both versions are equipped with all of the core visualization and editing features, but Pro offers additional online sharing functionality. Power BI Premium shares the same features as the Desktop Pro edition and allows users to use remote or on-premise server resources. Power BI also offers a free online service (Power BI Service), this allows the user to upload datasets or Power BI projects and use the online module to create visualizations with the data. However, Power BI Service limits computing resources by using a shared resource pool, whereas Power BI Premium allows the user to utilize dedicated, scalable resources for their group. 


\section{Developer Support}

This section covers the support features offered by each tool and the actions the developers take to ensure the product meets the customer's requests.

\subsection{Tableau}

Tableau offers a support page instead of a direct link to communicate with the developers, which allows the user to submit a case to contact support, download drivers, view known issues, etc. (Tableau Software Support n.d.). These requests are used to guide Tableau developers to fix issues, supply updates, and add new material to the different Tableau editions. Tableau makes it their goal to be in a constant state that strives to innovate and deliver new capabilities. As a result, Tableau updates multiple times each year to guarantee quality service to the user.

\subsection{JMP}

JMP is constantly evolving, innovating, and creating new and improved products. With updates being pushed constantly throughout the year, a JMP user can be confident that they are not using out-of-date software. JMP also offers a support page for the user to contact the developers directly, report problems, view new content from updates, read frequently asked questions, etc. (JMP Support Overview n.d.).

\subsection{Power BI}

The Power BI developers push an update each month to ensure that Power $\mathrm{BI}$ is up to the latest standards of business intelligence. In addition to consistent updates, the Power BI support page offers various tools to ensure the user can enjoy the product to its fullest capabilities. The Power BI support page contains references to video-guided learning lessons, documentation, sample dashboards, and community support forums. In addition, it offers a quick way to report any issue the user may have and a link to submit ideas directly to the developers (Microsoft Power BI Support n.d.). 


\section{Cross-Compatibility}

This section covers the compatibility of the various Operating System (OS) environments.

\subsection{Tableau}

Tableau supports Mac, Windows, Linux, and a mobile application for iOS and Android.

\subsection{JMP}

JMP supports Mac and Windows. Also, JMP supports the ability to view and interact with JMP reports via a mobile device.

\subsection{Power BI}

Power BI only supports Windows machines, however, the web version is compatible with Microsoft Edge, Internet Explorer 11, Chrome, Safari, and Firefox. Power BI also has a mobile application for iOS and Android. 


\section{Efficiency with Large Data}

For this section, a 2.3 Gigabyte (GB) comma-separated variable (csv) file with 800,000 rows and 150 columns was populated with randomlygenerated integer values. This file was used to test the size limitations on the desktop editions for each of the researched tools. These were all tested on a machine running Windows 10 with 16 GB of Random Access Memory (RAM), Intel(R) Core $^{\mathrm{TM}}$ i7-6820HQ central processing unit (CPU) @ 2.70 Gigahertz (GHz).

\subsection{Tableau}

Tableau was able to use the selected csv file instantaneously, however, it was unclear on how Tableau read in the data; whether it read the entire file into memory, or used optimization techniques to query specific data elements as they were needed in the visualizations. Once the file was selected, Tableau was able to quickly construct visualizations. With basic visualization types, such as scatter plots and histograms, Tableau was able to quickly update with varying parameter selections. However, once sub plotting techniques and additional parameters were introduced, Tableau's rendering capabilities became sluggish.

\subsection{JMP}

JMP was capable of loading in the test dataset, but due to the size of the file, this process took approximately three minutes. Once JMP successfully completed reading in the file, it was able to quickly render most of the visualizations offered. However, some rendering time was significantly impacted due to the magnitude of the calculations needed to create the visualizations.

\subsection{Power BI}

Power BI loads the entire dataset at once before visualizations can be created. With this dataset, Power BI took approximately five minutes for the entire dataset to be fully loaded in. Once it was ready, data visualizations could be made with ease. However, some visualizations, such as the scatter plot, quickly began using a considerable amount of the $\mathrm{CPU}$. The program became slow while rendering the plots and began to take longer as more columns were added. 


\section{Conclusion}

The purpose of this report is to address the customer's data analysis and visualization requirements. The team chose Tableau, JMP, and Power BI as the basis of this report in order to see how well they compare within the following selected parameters: cost, security, variety and customizability, documentation support, web-enabled vs. standalone, developer support, cross-compatibility, and efficiency with large data. The goal of this report is not to claim that one tool is better than another, but rather to inform customers of potential solutions by highlighting the advantages and disadvantages of the tools. Based on the results, this report successfully shows the strengths and weaknesses of the tools, and aims to ease the decision-making process of customers looking to make use of readily available software. 


\section{References}

Iseminger, D. M. Blythe, A. Saxton, T. Petersen, JiayueHu, and B. Hamilton. 2018. Power BI Security - Power BI. Microsoft Docs. Accessed 15 July 2018

https://docs.microsoft.com/en-us/power-bi/service-admin-power-bi-security.

Jain, Y. 2017. What is Tableau: Overview, application \& differentiation from Excel. Newgenapps. 06 October 2017. https://www.newgenapps.com/blog/what-is-tableauoverview-application-tableau-vs-excel.JMP Multichannel Marketing. nd. JMP Multichannel marketing: What it is and why it matters | SAS. Accessed 24 July 2018. https://www.sas.com/jmpstore/software/jmp/prodJMP.html.

JMP Predictive Analytics Software. nd. Multiple linear regression. 15 July 2018. https://www.jmp.com/en_us/software/predictive-analytics-software.html.

JMP Pro Multichannel marketing. n.d. What it is and why it matters | SAS. Accessed 24 July 2018. https://www.sas.com/jmpstore/products-solutions/jmp-pro/prodJMPPRO.html.

JMP Statistical Discovery. nd. Data analysis software, multiple linear regression. Accessed 15 July 2018. https://www.jmp.com/en us/software/data-analysissoftware.html.

JMP Support Overview. nd. Multiple Linear Regression. Accessed 16 July 2018. https://www.jmp.com/en_us/support.html.

Microsoft Bring your Data to Life. nd. Power BI | Interactive Data Visualization BI Tools. Accessed o6 August 2018. https://powerbi.microsoft.com/en-us/power-bi-pro/.

Microsoft Power BI Support. nd. Power BI | Interactive Data Visualization BI Tools. Accessed 16 July 2018. https://powerbi.microsoft.com/en-us/support/.

Microsoft Pricing for Power BI. nd. Microsoft Power BI | Interactive Data Visualization BI Tools. Accessed 15 July 2018. https://powerbi.microsoft.com/en-us/pricing/.

Power BI Premium calculator. nd. Power BI | Interactive Data Visualization BI Tools. Accessed 19 June 2018. https://powerbi.microsoft.com/en-us/calculator/.

Tableau Software Platform Security. nd. Platform Security. Accessed 15 July 2018. https://www.tableau.com/enterprise-it/security\#bCBDglTHQy08k5bh.99.

Tableau Software Pricing. nd. Pricing for data people. Accessed: 11 July 2018. https://www.tableau.com/pricing/teams-orgs.

Tableau Software Support. nd. Support and Services. Accessed 15 July 2018. https://www.tableau.com/support. 


\section{Appendix A: Comparison Matrix}

The comparison matrix (Table A1) is a graphical representation designed to show a brief overview of the results. The columns represent the tools being compared, whereas the rows represent the categories analyzed in each tool. Additional information about each category is discussed in the report.

Table A1. Comparison matrix of the data.

\begin{tabular}{|c|c|c|c|}
\hline & SAS JMP & Tableau & Microsoft Power BI \\
\hline Usability & Complex & Simple & Simple \\
\hline Cost & $\begin{array}{l}\text { Standard: } \$ 1785.00 \text { / } \\
\text { year } \\
\text { Pro: } \$ 14900.00 \text { / year }\end{array}$ & $\begin{array}{l}\text { Viewer: } \$ 144.00 \text { / year } \\
\text { Explorer: } \$ 420.00 \text { / year } \\
\text { Creator: } \$ 840.00 \text { / year }\end{array}$ & $\begin{array}{l}\text { Desktop: Free } \\
\text { Pro: } \$ 119.88 \text { / year } \\
\text { Premium: } \$ 599940.00 \text { / } \\
\text { Node / year }\end{array}$ \\
\hline Security & $\begin{array}{l}\text { Desktop: Local Device } \\
\text { Server: N/A }\end{array}$ & $\begin{array}{l}\text { Desktop: Local Device } \\
\text { Server: Industry Standard }\end{array}$ & $\begin{array}{l}\text { Desktop: Local Device } \\
\text { Server: Industry Standard }\end{array}$ \\
\hline Visualization Variety & Offers both 2D and 3D & Offers only 2D & Offers 2D and Limited 3D \\
\hline Customizability & $\begin{array}{l}\text { Can use JSL to execute } \\
\text { MATLAB, SAS, and R code }\end{array}$ & Supports R and Python & $\begin{array}{l}\text { Supports R and } \\
\text { community-supported }\end{array}$ \\
\hline $\begin{array}{c}\text { Documentation } \\
\text { Support }\end{array}$ & $\begin{array}{l}\text { Official and community- } \\
\text { supported }\end{array}$ & $\begin{array}{l}\text { Official and community- } \\
\text { supported }\end{array}$ & $\begin{array}{l}\text { Official and community- } \\
\text { supported }\end{array}$ \\
\hline $\begin{array}{c}\begin{array}{c}\text { Web-enabled } \\
\text { (Server) }\end{array} \\
\end{array}$ & No & Yes & Yes \\
\hline Desktop & Yes & Yes & Yes \\
\hline Developer Support & Yes & Yes & Yes \\
\hline Cross-Compatibility & Windows and Mac & $\begin{array}{l}\text { Mac, Windows, Linux, iOS, } \\
\text { and Android }\end{array}$ & Windows, iOS, and Android \\
\hline $\begin{array}{r}\text { Efficiency with } \\
\text { Large Data }\end{array}$ & $\begin{array}{l}\text { Tested and worked with a } \\
2.3 \mathrm{~GB} \text {, randomly- } \\
\text { generated csv flat file }\end{array}$ & $\begin{array}{l}\text { Tested and worked with a } \\
2.3 \mathrm{~GB} \text {, randomly- } \\
\text { generated csv flat file }\end{array}$ & $\begin{array}{l}\text { Tested and worked with a } \\
2.3 \mathrm{~GB} \text {, randomly- } \\
\text { generated csv flat file }\end{array}$ \\
\hline
\end{tabular}




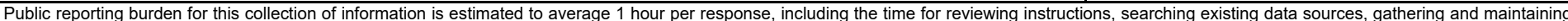

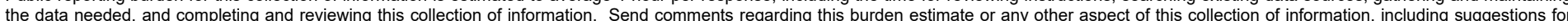

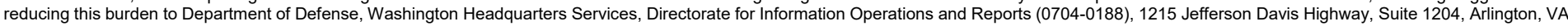

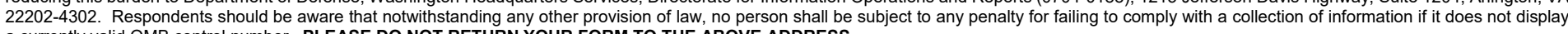
a currently valid OMB control number. PLEASE DO NOT RETURN YOUR FORM TO THE ABOVE ADDRESS.
1. REPORT DATE $(D D-M M-Y Y Y Y)$
August 2019
2. REPORT TYPE
Final report

3. DATES COVERED (From - To)

\section{TITLE AND SUBTITLE}

TradeAnalysis Visualization Comparisons

5a. CONTRACT NUMBER

5b. GRANT NUMBER

5c. PROGRAM ELEMENT NUMBER

\section{AUTHOR(S)}

Kenneth D. Niles, Joseph E. Jabour, Joshua Q. Church, and Timothy W. Garton

5d. PROJECT NUMBER

5e. TASK NUMBER

5f. WORK UNIT NUMBER

92L5D8

\section{PERFORMING ORGANIZATION NAME(S) AND ADDRESS(ES)}

8. PERFORMING ORGANIZATION REPORT NUMBER

U.S. Army Engineer Research and Development Center,

Information Technology Laboratory

ERDC/ITL SR-19-15

3909 Halls Ferry Road, Vicksburg, MS 39180-6199

9. SPONSORING / MONITORING AGENCY NAME(S) AND ADDRESS(ES)

10. SPONSOR/MONITOR'S ACRONYM(S)

Headquarters, U.S. Army Corps of Engineers

Washington, DC 20314-1000

11. SPONSOR/MONITOR'S REPORT NUMBER(S)

\section{DISTRIBUTION / AVAILABILITY STATEMENT}

Approved for public release; distribution unlimited.

\section{SUPPLEMENTARY NOTES}

\section{ABSTRACT}

The objective of this report is to identify available tools that satisfy the customer's needs instead of recreating a tool that already exists. With the vast amount of data analysis and visualization tools on the market, it can be difficult to choose the correct tool for any given purpose. It should be noted that the goal of this report is not to determine the best data analysis and visualization tool, but rather to inform readers about specific factors each tool offers. The TradeAnalysis team (in support of the Engineered Resilient Systems Decision Support Tradespace Analytics team) achieved this goal by first creating a list of factors to objectively compare each tool. Once the factors were determined, each team member was tasked to find viable tools on the market capable of generalizing solutions to customer's requirements. Once the research was done, the team gathered the data and put together this report to compare the advantages and disadvantages of the tools.

\begin{tabular}{|lll}
\hline 15. SUBJECT TERMS & Systems engineering--Decision making & Quantitative research \\
& Big data & Computer programs \\
& Information visualization &
\end{tabular}

16. SECURITY CLASSIFICATION OF:

\begin{tabular}{|l|l|}
\hline a. REPORT & b. ABSTRACT \\
UNCLASSIFIED & UNCLASSIFIED
\end{tabular}

17. LIMITATION OF ABSTRACT

18. NUMBER
OF PAGES
27

19a. NAME OF RESPONSIBLE PERSON

19b. TELEPHONE NUMBER (include area code) 